\title{
Stable smectic phase in suspensions of polydisperse colloidal platelets with identical thickness
}

\author{
Dazhi Sun and Hung-Jue Sue \\ Department of Mechanical Engineering, Texas A\&GM University, College Station, TX 77843 USA. \\ Zhengdong Cheng* \\ Artie McFerrin Department of Chemical Engineering, \\ Texas A\& M University, College Station, TX 77843 USA. \\ Yuri Martínez-Ratón才 \\ Grupo Interdisciplinar de Sistemas Complejos (GISC), Departamento de Matemáticas, \\ Escuela Politécnica Superior, Universidad Carlos III de Madrid, \\ Avenida de la Universidad 30, E-28911, Leganés, Madrid, Spain \\ Enrique Velasco \\ Departamento de Física Teórica de la Materia Condensada and Instituto de Ciencia de Materiales Nicolás Cabrera, \\ Universidad Autónoma de Madrid, E-28049 Madrid, Spain. \\ (Dated: September 14, 2009)
}

\begin{abstract}
We report the nematic and smectic ordering in a new aqueous suspension of monolayer $\alpha$ Zirconium phosphate platelets possessing a high polydispersity in diameter but uniform thickness. We observe an isotropic-nematic transition as the platelet volume fraction increases, followed by the formation of a smectic, an elusive phase that has been rarely seen in discotic liquid crystals. The smectic phase is characterized by X-ray diffraction, high-resolution transmission electron microscopy, and optical microscopy. The phase equilibria in this highly polydisperse suspension is rationalized in terms of a theoretical approach based on density-functional theory.
\end{abstract}

PACS numbers: 61.30.Eb, 64.70.M-, 81.16.Dn, 82.70.Dd

Synthetic inorganic platelets are being used extensively to investigate phase transitions in discotic liquid crystals consisting of colloidal, approximately disc-shaped, particles in aqueous suspension. Starting from the completely disordered isotropic (I) phase in the dilute regime, most discotic suspensions exhibit an orientationally ordered nematic $(\mathrm{N})$ phase as the volume fraction of the platelets in the sample increases. At higher volume fractions a columnar (C) phase, with columns of platelets arranged in a two-dimensional lattice, has been observed in some materials, either directly from the I phase [1] or via N ordering [2]. Computer simulations on monodisperse [3] and polydisperse [4] hard platelets of high aspect ratio show that indeed these liquid-crystalline phases can be stabilized solely from exclusion interactions, but no layered ordering, of smectic (S) type, is found. However, the work of van der Kooij et al. 2] seemed to indicate that smectic or lamellar phases might exist in colloidal platelet suspensions at high volume fractions. Usually these suspensions are polydisperse in both thickness and diameter. The $\mathrm{C}$ phase stabilizes even for a considerable degree of diameter polydispersity, but uniformly thick platelets with very high diameter polydispersity cannot form a $\mathrm{C}$ phase, which presumably would be superseded by a $\mathrm{S}$

\footnotetext{
*Electronic address: cheng@chemail.tamu.edu

${ }^{\dagger}$ Electronic address: yuri@math.uc3m.es
}

phase before the glassy or crystalline phases. Nevertheless, the $\mathrm{S}$ phase remains elusive [2] and swollen lamellar phases has been observed to form, directly from a I suspension, only in a few materials $[\underline{5}, \underline{6}]$.
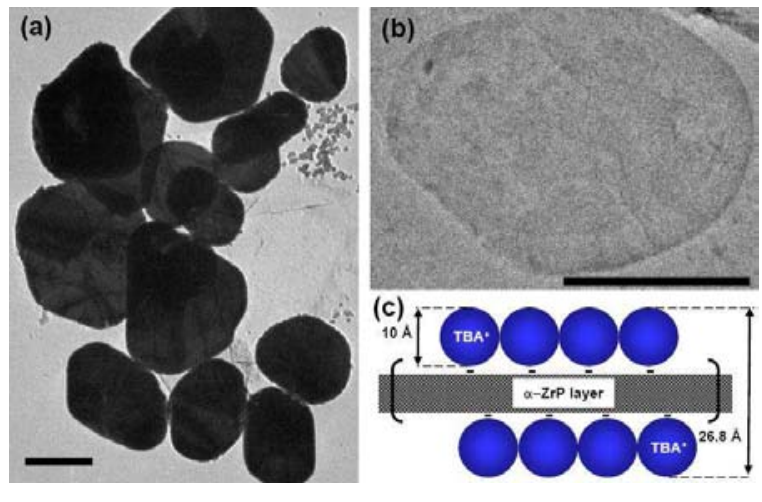

FIG. 1: (Color online). $\alpha$-Zirconium phosphate platelets. (a) TEM of pristine ZrP platelets. (b) TEM of a ZrP monolayer platelet exfoliated by TBA. (c) Thickness schematic of a ZrP monolayer platelet. Length is not drawn to scale. The scale bars in (a) and (b) are $1 \mu \mathrm{m}$.

Here we report the observation of a stable $\mathrm{S}$ phase in a new system of platelets with high polydispersity in diameter but identical thickness. Contrary to the approximately hard-core platelets analyzed in Ref. 2], our particles interact via long-range forces. We find that, as the platelet volume fraction $\phi$ increases, the samples fol- 
low the phase sequence $\mathrm{I}-\mathrm{N}-\mathrm{S}$, with no stable $\mathrm{C}$ phase in the volume-fraction interval explored. Some features of the phase transitions can be explained in terms of a density-functional theory (DFT) for a simple interaction model.

Pristine $\alpha$-Zirconium phosphate (ZrP) layered platelets were synthesized through a hydrothermal method similar to that reported in Ref. [7] and exfoliated with tetra-n-butylammonium hydroxide (TBA ${ }^{+} \mathrm{OH}^{-}$, Aldrich, $1 \mathrm{~mol} / \mathrm{l}$ in methanol) at a molar ratio of ZrP:TBA = 1:1 in de-ionized water. No salts were added during the experiments. After reaction, the platelets were allowed to expose in sonication for 5 mins and then sit for one day to reach phase transition equilibrium. The platelets, which contain two TBA monolayers, have an identical thickness of $L=2.68 \mathrm{~nm}$. This process has been demonstrated [7] to lead to stable aqueous colloidal suspensions (see Fig. 1). The average platelet is cylindrical but with relatively high variations in transverse shape. Samples containing various platelet volume fractions but the same polydispersity were prepared. Transmission Electron Microscopy (TEM) images [Fig. 1(b)] allow for an approximate estimation of mean particle diameter $(\sim 2 \mu \mathrm{m})$.

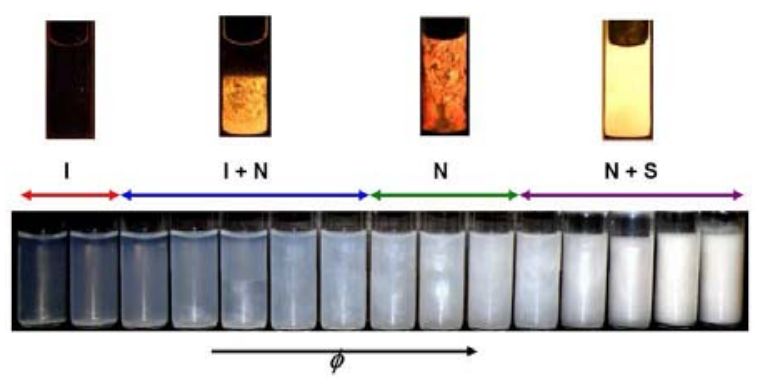

FIG. 2: (Color online). Optical image (bottom) and images under cross polarizers (top) of samples. Bottom sequence corresponds to platelet volume fractions (from left to right) $\phi=0.004,0.007,0.009,0.013,0.017,0.021,0.031,0.041$, $0.048,0.054,0.059,0.074,0.100,0.160$, and 0.200 . Range of phase stability and phase coexistence are indicated by double headed arrows.

To obtain a more accurate estimation of mean platelet diameter and polydispersity, Dynamic Light Scattering (DLS) was performed on the exfoliated $\mathrm{ZrP}$ monolayer platelets, yielding a platelet diameter of $2295 \mathrm{~nm}$ (close to the TEM estimation) and a polydispersity $\sigma_{\mathrm{D}}=32 \%$. To search for possible spatial ordering of the platelets, aqueous suspensions were directly characterized by X-ray diffraction (XRD).

At $\phi \lesssim 0.01$, the platelet suspension forms a random stable dispersion in water (I phase). For samples with high $\phi$, birefringent features through crossed polarizers (Fig. 2) reveal the occurrence of a transition to a nematic phase for $\phi \gtrsim 0.01$. In the interval $0.01 \lesssim \phi \lesssim 0.03$, I and $\mathrm{N}$ phases coexist. A full nematic phase is obtained for $0.03 \lesssim \phi \lesssim 0.06$. The macroscopic phase separa-
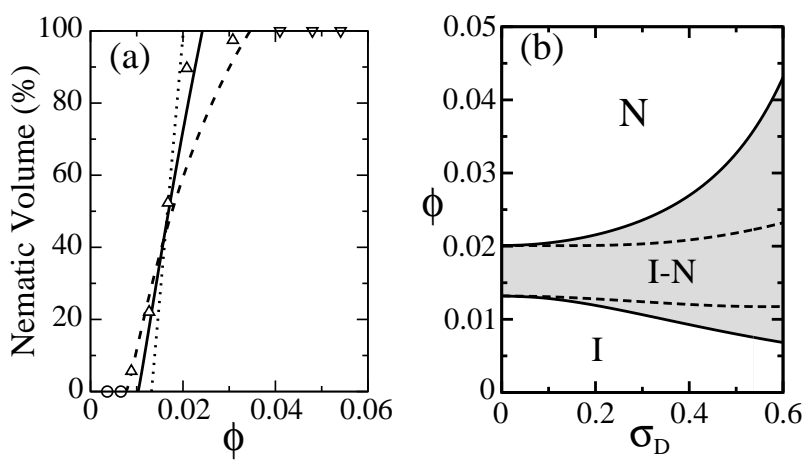

FIG. 3: (a) Nematic volume fraction (sample volume occupied by the $\mathrm{N}$ phase) as a function of platelet volume fraction. Symbols: experimental results. Lines: theoretical results with $\sigma_{\mathrm{D}}=0.00$ (dotted), 0.32 (continuous), and 0.52 (dashed). (b) Theoretical platelet volume fraction as a function of polydispersity for the I-N transition. Solid lines: I and $\mathrm{N}$ cloud curves. Dashed lines: I and $\mathrm{N}$ shadow curves. The coexistence region is shaded.

tion is completed within 24 hours. Fig. 3 shows how the fraction of total sample volume occupied by the $\mathrm{N}$ phase behaves as a function of $\phi$ (symbols). Nonlinearity in the two-phase region is due to fractionation effects: the diameter polydispersity of the platelets in the I and $\mathrm{N}$ phases are different (monodisperse suspensions would follow the lever rule and exhibit linear behavior).

At high volume fraction of platelets, multiple scattering peaks up to 5 orders in XRD experiments are observed (Fig. 4) when $\phi>0.06$. These scattering peaks are the (001) family of layered arrangements of colloidal platelets (S phase). No XRD peaks were obtained for $\phi<0.06$ (I or $\mathrm{N}$ phases). The $\mathrm{S}$ phase has been frequently observed in liquid crystals of rod-like molecules with layered structure. However, these organic smectic phases normally lack high-order XRD peaks due to their small domain size. For our colloidal platelets, since the size of the mesogen is of the order of hundreds of nanometers to microns, the domain size can easily reach tens of microns to millimeters. The presence of higherorder diffraction peaks and their relatively strong intensity (Fig. 4) is the result of the large domain size. Fig. 5 shows the optical microscopy image of the smectic liquid crystal observed in our system. A ribbon-like smectic mesophase around $50 \mu \mathrm{m}$ wide and with a length of up to a few millimeters has been observed. Considering the size of the plate mesogens (around $2 \mu \mathrm{m}$, Fig. 1), the optical microscopy image clearly demonstrates the assembly of our colloidal platelets into smectic layers parallel to the the plane of the image.

Unfortunately it was not possible to directly measure the nematic, $\phi_{N}$, and smectic, $\phi_{S}$, volume fractions (i.e. fractions of volume occupied by platelets in the respective phase). An upper limit for $\phi_{S}$ can be roughly estimated by assuming smectic layers to consist of single platelet sheets in a random close-packed arrange- 


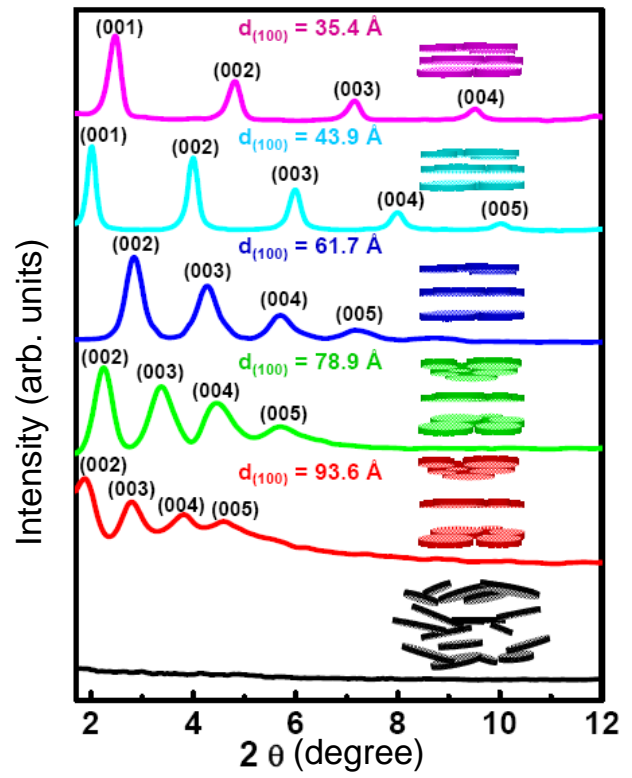

FIG. 4: (Color online). XRD intensities as a function of scattering angle for various volume fractions. From top to bottom: $\phi=0.200,0.160,0.100,0.074,0.059$, and 0.054 .

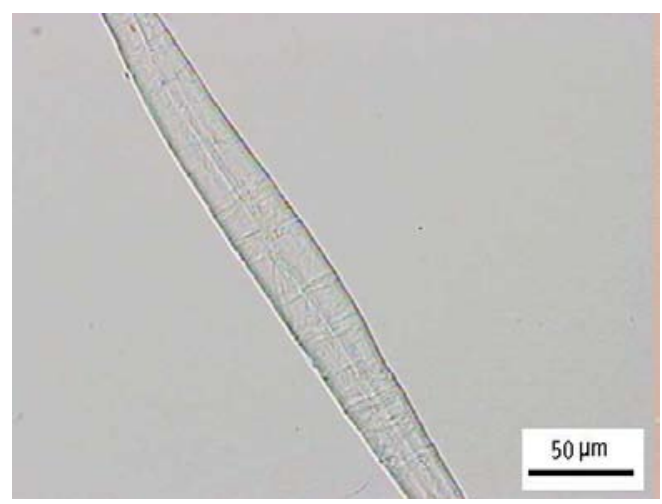

FIG. 5: Optical microscopy image of the smectic domains.

ment, which gives $\phi_{S}=\phi_{2 D} L / d, \phi_{2 D}=0.815$ being the random close-packing area fraction of polydisperse hard disks with $\sigma_{\mathrm{D}}=0.3$ [8], and $d$ the smectic period. In Table \values of $\phi_{S}$ are collected under the column labeled max.

In the experiments the $\mathrm{S}$ phase occupies a small, slowly expanding, region of the sample. It seems that the phase transition (which may take many days to complete due to the high viscosity of the samples) is not proceeding fast enough to complete within the finite observation time. The weak diffraction peaks in the XRD spectra for the first smectic state lend support to a weakly first-order or continuous scenario for the $\mathrm{N}-\mathrm{S}$ transition.

One interesting feature is that the layer spacing (Table I) exhibits a large variation (close to three-fold) in the volume-fraction interval explored, which may point to the existence of long-range platelet repulsion due
TABLE I: Characteristics of the smectic phase measured in the experiments. $\Delta \phi=\phi-\phi^{* \exp }$, with $\phi^{* \exp } \simeq 0.059$ the estimated experimental volume fraction at the spinodal point.

\begin{tabular}{cccccc}
\hline \hline$\phi$ & $\Delta \phi$ & $d(\mathrm{~nm})$ & $d / L$ & $L_{\text {eff }} / L$ & $\begin{array}{c}\phi_{S} \\
\text { estim. (max.) }\end{array}$ \\
\hline 0.059 & 0.000 & 9.36 & 3.49 & 2.67 & $0.108(0.233)$ \\
0.074 & 0.015 & 7.89 & 2.94 & 2.27 & $0.136(0.277)$ \\
0.102 & 0.043 & 6.17 & 2.30 & 1.80 & $0.193(0.354)$ \\
0.161 & 0.102 & 4.39 & 1.64 & 1.34 & $0.326(0.497)$ \\
0.199 & 0.140 & 3.54 & 1.32 & 1.11 & $0.441(0.617)$ \\
\hline \hline
\end{tabular}

to platelet surface charges or possibly hydration forces (since platelets are hydrophilic, water-mediated effects might be at work [9]). Attractive van der Waals forces may also operate at shorter distances.

To rationalize these findings, we map the real particle onto an effective model system of parallel polydisperse hard cylinders of diameter $D$ and thickness $L_{\text {eff }}>L$. Essentially, the model incorporates long-range repulsion via an effective particle thickness (a procedure used to model aqueous suspensions of charged virus particles with added salt [10]). For the moment we simply note that the value of $L_{\text {eff }}$ will depend on $\phi$ and the type of phase.

We obtained some predictions for the hard-core model by applying a theoretical analysis based on DFT. In the case of the I-N transition we used Onsager theory [11], which takes into account excluded-volume effects at second order in density and should give accurate results because of the high diameter-to-thickness ratio of the platelets. Since samples are polydisperse, a given distribution of platelet diameters has to be imposed. Furthermore, the nematic order parameter, measuring the degree of orientation of the particle main axis about the director, is expected to be very high and the simplifying Zwanzig approximation [12] (where only three mutually perpendicular particle orientations, along $x, y$ and $z$ Cartesian axes, are permitted) can be implemented. The total free-energy density in reduced thermal units $\left(k_{B} T\right)$, $\Phi=\Phi_{\mathrm{id}}+\Phi_{\text {exc }}$, can be split into ideal,

$\Phi_{\mathrm{id}}=\int d r\left\{2 \rho_{\perp}(r)\left[\ln \rho_{\perp}(r)-1\right]+\rho_{\|}(r)\left[\ln \rho_{\|}(r)-1\right]\right\}$,

and excess

$$
\Phi_{\mathrm{exc}}\left(\pi\langle D\rangle^{3} / 4\right)=2\left[m_{\perp}^{(1)} m_{\perp}^{(2)}+m_{\|}^{(1)} m_{\perp}^{(2)}+m_{\|}^{(2)} m_{\perp}^{(1)}\right],
$$

parts. The moments $m_{\mu}^{(\alpha)}$ of the number density distribution functions $\rho_{\mu}(r)$ are defined as

$$
m_{\mu}^{(\alpha)}=\left(\pi\langle D\rangle^{3} / 4\right) \int d r r^{\alpha} \rho_{\mu}(r) .
$$

We use the dimensionless platelet diameter $r=D /\langle D\rangle$ (with $\langle D\rangle$ the mean diameter) as polydispersity variable, and used the notation $\rho_{x}(r)=\rho_{y}(r)=\rho_{\perp}(r)$ and 
$\rho_{z}(r)=\rho_{\|}(r)$ to label the densities of species oriented perpendicular and parallel to the director, respectively. For the I and $\mathrm{N}$ symmetries we have

$$
\begin{aligned}
& \rho_{\perp}^{\mathrm{I}}(r)=\rho_{\|}^{\mathrm{I}}(r)=\frac{\rho^{\mathrm{I}}(r)}{3}, \\
& \rho_{\perp}^{\mathrm{N}}(r)=\frac{1-Q(r)}{3} \rho^{\mathrm{N}}(r), \quad \rho_{\|}^{\mathrm{N}}(r)=\frac{1+2 Q(r)}{3} \rho^{\mathrm{N}}(r),
\end{aligned}
$$

where $-1 / 2 \leq Q(r) \leq 1$ is the $\mathrm{N}$ order parameter of species with reduced diameter $r$. To find the I and $\mathrm{N}$ coexistence densities, i.e.

$$
\rho^{\mathrm{I}, \mathrm{N}}=\sum_{\mu=x, y, z} \int d r \rho_{\mu}^{\mathrm{I}, \mathrm{N}}(r)
$$

in a system with a relative fraction of volume occupied by the $\mathrm{N}$ phase given by the parameter $0 \leq \gamma \leq 1$, we need to implement the constrained functional minimization of

$$
\Phi=(1-\gamma) \Phi^{\mathrm{I}}+\gamma \Phi^{\mathrm{N}},
$$

with respect to $\rho_{\mu}^{\mathrm{I}, \mathrm{N}}(r) . \quad \Phi^{I, N}$ are the total free-energy densities of the coexisting I and $\mathrm{N}$ phases. The constraint is dictated by the lever rule restriction:

$$
\rho_{0}(r)=(1-\gamma) \rho^{\mathrm{I}}(r)+\gamma \rho^{\mathrm{N}}(r),
$$

where $\rho_{0}(r)=\rho_{0} h(r)$ is the parent number density distribution function with a fixed function $h(r)$, which is the diameter distribution function density normalized as $\int d r h(r)=1$. We choose a two-parameter function which, after normalising and applying the additional condition $\int \operatorname{drrh}(r)=1$ (note that this is equivalent to $\langle r\rangle=1)$, becomes

$h(r)=\frac{2 \Gamma[(\nu+2) / 2]^{\nu+1}}{\Gamma[(\nu+1) / 2]^{\nu+2}} r^{\nu} \exp \left\{-\left(\frac{\Gamma[(\nu+2) / 2]}{\Gamma[(\nu+1) / 2]} r\right)^{2}\right\}$,

where $\Gamma(x)$ is the Gamma function. The free parameter $\nu>0$ controls the degree of polydispersity. Note that the present function $h(r)$ has a Gaussian tail for $r \gg 1$, while it fulfills $h(0)=0$, in contrast to the Gaussian distribution function. The constrained minimization of Eq. (77) and the equality of osmotic pressures

$$
\beta P^{\mathrm{I}, \mathrm{N}}=\rho^{\mathrm{I}, \mathrm{N}}+\Phi_{\text {exc }}^{\mathrm{I}, \mathrm{N}}
$$

between both phases allow us to find the coexistence total densities as a function of $\gamma$. For $\gamma=0$ we find the coexistence between a I phase which occupies the entire volume, called the cloud phase, and an infinitesimal amount of the $\mathrm{N}$ phase (called shadow phase). In the other limit, $\gamma=1$, we find the cloud N-shadow I coexistence. Results for the I-N transition are plotted in Fig. 6(a) as lines for three different polydispersities: $\sigma_{\mathrm{D}}=0.00$ (monodisperse fluid), 0.32 (same as in experiment), and 0.52. The effective thickness (assumed to be independent of $\phi$ ) was
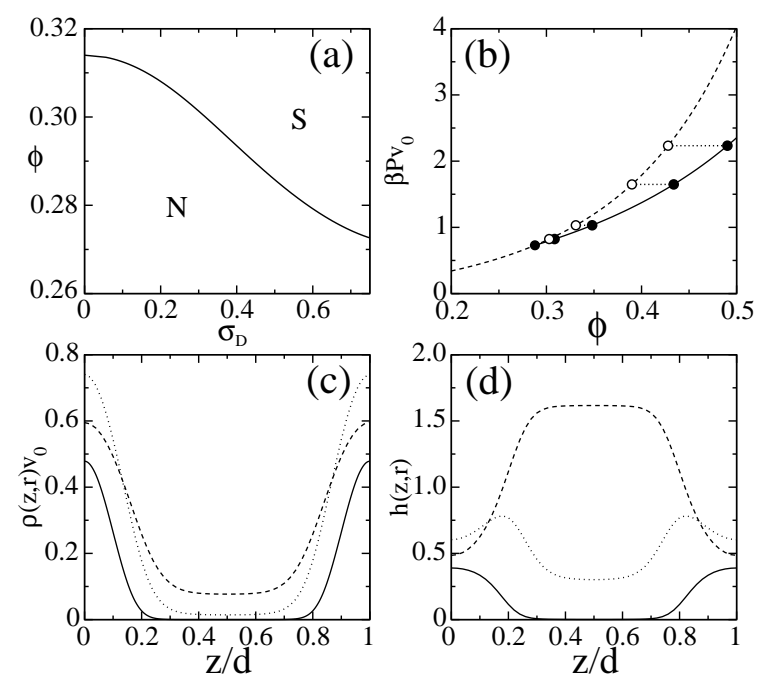

FIG. 6: (a) Theoretical volume fraction of the spinodal instability against smectic fluctuations as a function of polydispersity. (b) Osmotic pressure (in reduced units) vs. platelet volume fraction $\phi$ for smectic (continuous line) and nematic (dashed line) for $\sigma_{\mathrm{D}}=0.52$. Filled circles: smectic states; open circles: unstable nematic states with $\phi$ obtained as $\phi=\phi^{* \text { the }}+\Delta \phi$, with $\phi^{* \text { the }}=0.288$ (theoretical bifurcation point) and $\Delta \phi$ taken from experiment (see Table I). (c) Density profile $\rho(z, r)$ for $r=1.5$ (solid line), 0.4 (dashed) and 0.8 (dotted). (d) Normalized density distribution function $h(z, r)$ in one smectic period. Lines as in panel (c). In (c) and (d) the smectic volume fraction is $\phi_{S}=0.452$ and the smectic period $d / L_{\text {eff }}=1.211$.

set to $L_{\text {eff }}=9 L$ so as to make the experimental and theoretical curves with the same value of $\sigma_{\mathrm{D}}$ approximately overlap (note however that our theoretical data, without scaling, agree well with the experimental results of van der Kooij et al. [2] for their approximately hard platelets). We note that: (i) for high $\sigma_{\mathrm{D}}$ the curvature in the transition region is similar to that of the experiment. (ii) The I+N coexistence gap is too small in the theory; this may be due to the crudeness of the model, and/or to an inaccurate experimental estimation of $\sigma_{\mathrm{D}}$ (note that the width of the $\mathrm{I}+\mathrm{N}$ region in the theoretical curve for $\sigma_{\mathrm{D}}=0.52$ is quite close to the experimental one). As inferred from Fig. 3(b), the coexistence gap (defined as the distance between the $\mathrm{I}$ and $\mathrm{N}$ cloud curves at the same polydispersity) increases with $\sigma_{\mathrm{D}}$ due to fractionation effects: larger platelets tend to go to the $\mathrm{N}$ phase, while smaller platelets preferentially populate the I phase.

Next we turn to the theoretical description of the N-S transition. The $\mathrm{S}$ phase demands a description in terms of the density distribution $\rho(z, r)$, where $z$ is the distance along the nematic director. From this, the local smectic volume fraction can be calculated, $\phi_{S}(z)=$ $v_{0} \int d r r^{2} \rho(z, r)$ (with $v_{0}=\pi\langle D\rangle^{2} L_{\text {eff }} / 4$ an average particle volume), and also a mean smectic volume fraction, $\phi_{S}=d^{-1} \int_{0}^{d} d z \phi_{S}(z)$. A DFT fundamental-measure scheme for strictly parallel hard cylinders [13] was gener- 
alized to the polydisperse case (the assumption of parallel particles should be valid since the nematic order parameter is expected to be very high). The excess, $z$-dependent local free-energy density in units of $k_{B} T$ is

$$
\Phi_{\text {exc }} v_{0}=-n_{0} \ln \left(1-n_{3}\right)+\frac{2 n_{1}^{\perp} n_{2}^{\perp}+n_{1}^{\|} n_{2}^{\|}}{1-n_{3}}+\frac{n_{2}^{\|}\left(n_{2}^{\perp}\right)^{2}}{\left(1-n_{3}\right)^{2}},
$$

where the weighted densities are

$$
\begin{array}{ll}
n_{0}(z)=\mathcal{M}^{(0)} * \omega^{(0)}(z), & n_{3}(z)=\mathcal{M}^{(2)} * \omega^{(3)}(z), \\
n_{1}^{\perp}(z)=\mathcal{M}^{(1)} * \omega^{(0)}(z), & n_{1}^{\|}(z)=\mathcal{M}^{(0)} * \omega^{(3)}(z), \\
n_{2}^{\perp}(z)=\mathcal{M}^{(1)} * \omega^{(3)}(z), & n_{2}^{\|}(z)=\mathcal{M}^{(2)} * \omega^{(0)}(z),
\end{array}
$$

with $\mathcal{M}^{(\alpha)}(z)=m_{\|}^{(\alpha)}(z) / \kappa$ and $\kappa=\langle D\rangle / L_{\text {eff }}$. The weights are

$$
\omega^{(0)}(z)=\frac{1}{2} \delta\left(\frac{1}{2}-|z|\right), \quad \omega^{(3)}(z)=\Theta\left(\frac{1}{2}-|z|\right),
$$

with $\delta(z)$ and $\Theta(z)$ the usual Dirac-delta and Heavisidestep functions; the symbol $*$ denotes convolution, i.e.

$$
\begin{aligned}
& f * \omega^{(0)}(z)=\frac{1}{2}[f(z-1 / 2)+f(z+1 / 2)], \\
& f * \omega^{(3)}(z)=\int_{z-1 / 2}^{z+1 / 2} d z^{\prime} f\left(z^{\prime}\right) .
\end{aligned}
$$

In all preeceding equations (12)-(14) the coordinate $z$ is in units of $L_{\text {eff }}$. The ideal and the interaction free-energy functionals per unit volume for the smectic are then

$$
\begin{aligned}
& \beta \mathcal{F}_{\text {id }} / V=d^{-1} \int_{0}^{d} d z \int d r \rho(z, r)[\ln \rho(z, r)-1], \\
& \beta \mathcal{F}_{\text {exc }} / V=d^{-1} \int_{0}^{d} d z \Phi_{\text {exc }}(z) .
\end{aligned}
$$

The equation of state of S phase was obtained by functional minimization of the total free-energy with respect to $\rho(z, r) \equiv \rho_{\|}(z, r)$ with the restriction $\rho_{0}(r)=$ $d^{-1} \int_{0}^{d} d z \rho(z, r), \rho_{0}(r)$ being the (fixed) mean density distribution function per period. The $\mathrm{N}-\mathrm{S}$ transition is found to be continuous for all values of polydispersity (see Fig. 6 (a), where the limit $\sigma_{\mathrm{D}}=0$ corresponds to the one-component model of Ref. 14]). This is in qualitative agreement with experiments which, as discussed above, suggest a continuous or weakly first-order phase transition [15].

Fig. 6(b) shows the osmotic pressure for $\mathrm{N}$ and $\mathrm{S}$ phases as a function of $\phi$ for the hard-core potential. The $\mathrm{N}$ phase is unstable beyond the bifurcation point. Even though there are no signs of $\mathrm{N}+\mathrm{S}$ coexistence or macroscopic fractionation at the transition, some subtle micro-fractionation effects in the $\mathrm{S}$ phase exist, shown in Figs. 66(c-d) which pertain to values $\sigma_{\mathrm{D}}=0.52$ and $\phi_{S}=0.452$. Density profiles for different diameters show micro-fractionation: the probability density $h(z, r)=\rho(z, r) / \int d r \rho(z, r)$ shows that the relative fraction of large platelets is higher at smectic layers, while the opposite occurs for smaller platelets, with maxima at interstitials. The relative fraction of intermediatelysized platelets has two maxima per period, located a bit off the layers.

Finally we extract some information from the model in order to interpret experimental data and, in particular, to refine the values for $\phi_{S}$ previously derived as upper estimates. We assume the experimental transition to occur at a platelet volume fraction $\phi^{*} \approx 0.059$ (interpreted as a spinodal point or as the point where the smectic phase appears for the first time). The experimentally observed $\mathrm{N}$ states for $\phi \gtrsim \phi^{*}$ would then be unstable, with nematic volume fractions $\phi_{N}<\phi_{S}$; here $\phi_{S}$ is the (unknown) platelet volume fraction of the stable smectic state (at the same osmotic pressure). Since the smectic volume in the samples is small, we take $\phi_{N} \simeq \phi$. Also, we identify the (unstable) experimental nematic states with the theoretical (unstable) nematic branch. We predict $\phi_{S}$ from our equation of state, Fig. 6(b), as follows. First, the volume-fraction distance $\Delta \phi=\phi-\phi^{*}$ is taken to be the same in both theory and experiment, which allows to obtain, for each $\phi$ (or $\Delta \phi$ ) in Table【, a theoretical value $\phi_{S}^{\text {the }}$ [indicated by filled circles in Fig. [6(b)], to which our hard-core theory uniquely associates a value $d / L_{\text {eff }}$, and estimate $L_{\text {eff }} / L=(d / L) /\left(d / L_{\text {eff }}\right)$ (see Table Ii); from low to high volume fraction, $L_{\text {eff }} / L=2.67-1.11$. Now the estimated $\phi_{S}$ for the real system follows from $\phi_{S}=\phi_{S}^{\text {the }} /\left(L_{\text {eff }} / L\right)$-see Table I under column estim. [16]. Even though the refined procedure probably gives more reasonable values for $\phi_{S}$ than the previous crude estimates, we cannot at present improve the model for lack of accurate experimental measurements. However, all evidence points to the existence of a weak phase transition from a $\mathrm{N}$ to a stable $\mathrm{S}$ phase in this novel colloidal suspension of platelets.

To summarize, we have experimentally analyzed a new highly polydisperse colloidal platelet suspension exhibiting smectic ordering, thus confirming that this elusive phase can be stabilized in platelet colloids when polydispersity suppresses the columnar phase. A theoretical model for polydisperse platelets based on DFT and an effective hard-core model partially explains the experimentally observed phase behavior.

We thank J. A. Cuesta and A. Clearfield for useful discussions and a critical reading of the manuscript. Acknowledgment is made to the donors of ACS Petroleum Research Fund (45303-G7) and to the Dow Chemical Company. This work has been partly financed by start-up funds from Texas Engineering Experimental Station and Texas A\&M University, by grants NANOFLUID, MOSAICO and S-0505/ESP-0299 from Comunidad Autónoma de Madrid (Spain), and Grants 
FIS2005-05243-C02-01, FIS2007-65869-C03-01, FIS200805865-C02-02 and FIS2007-65869-C03-C01 from Ministe- rio de Educación y Ciencia (Spain).
[1] A. B. D. Brown, C. Ferrero, T. Narayanan, and A. R. Rennie, Eur. Phys. J. B 11, 481 (1999).

[2] F. M. van der Kooij, K. Kassapidou, and N. W. Lekkerkerker, Nature 406, 868 (2000).

[3] J. A. C. Veerman and D. Frenkel, Phys. Rev. A 45, 5632 (1992).

[4] M. Bates and D. Frenkel, J. Chem. Phys. 110, 6553 (1999).

[5] J. C. P. Gabriel, F. Camerel, B. J. Lemaire, H. Desvaux, P. Davidson, and P. Batail, Nature 413, 504 (2001).

[6] N. Wang, S. Liu, J. Zhang, Z. Wu, J. Chen, and D. Sun, Soft Matter 1, 428 (2005).

[7] L. Y. Sun, W. J. Boo, D. H. Sun, A. Clearfield, and H. J. Sue, Chem. Mater. 19, 1749 (2007); H. N. Kim, S. W. Keller, T. E. Mallouk, J. Schmitt, G. Decher, Chem. Mater. 9, 1414 (1997).

[8] R. Ogata, T. Odagaki and K. Okazaki, J. Phys.: Condens. Matter 17, 4531 (2005).

[9] J. N. Israelachvili in Intermolecular and Surface forces pp. 122-133, Academic Press, London (1992).
[10] Z. Dogic, Phys. Rev. Lett. 91, 165701 (2003).

[11] L. Onsager, Ann. N. Y. Acad. Sci. 51, 627 (1949).

[12] R. W. Zwanzig, J. Chem. Phys. 24, 855 (1956); 39, 1714 (1963).

[13] Y. Martínez-Ratón, J. A. Capitán and J. A. Cuesta, Phys. Rev. E 77, 051205 (2008).

[14] J. A. Capitán, Y. Martínez-Ratón and J. A. Cuesta, J. Chem. Phys. 128, 194901 (2008).

[15] Further theoretical calculations with attractive forces (not shown) indicate that the transition changes to first order when the system is polydisperse (orientational degrees of freedom might have some additional effect on the transition).

[16] Note that the smectic volume fraction $\phi_{S}^{\text {the }}$ and period $d / L_{\text {eff }}$ at the spinodal point could both decrease due to additional attractive, short-ranged forces plus longranged, repulsive forces; in this case $L_{\text {eff }} / L$ would increase and consequently $\phi_{S}$ would be lower than our estimate (0.108) using a purely repulsive potential. 